Archives

$5 \mid 1990$

Varia

\title{
L'américanisation de la France (1945-1970)
}

\section{Richard Kuisel}

\section{(2) OpenEdition}

\section{Journals}

Édition électronique

URL : http://journals.openedition.org/ccrh/2889

DOI : $10.4000 /$ ccrh.2889

ISSN : 1760-7906

Éditeur

Centre de recherches historiques - EHESS

Édition imprimée

Date de publication : 15 avril 1990

ISSN : 0990-9141

Référence électronique

Richard Kuisel, «L'américanisation de la France (1945-1970) », Les Cahiers du Centre de Recherches Historiques [En ligne], 5 | 1990, mis en ligne le 20 mars 2009, consulté le 19 avril 2019. URL : http:// journals.openedition.org/ccrh/2889; DOI : 10.4000/ccrh.2889

Ce document a été généré automatiquement le 19 avril 2019

Article L.111-1 du Code de la propriété intellectuelle. 


\title{
L'américanisation de la France (1945-1970)
}

\author{
Richard Kuisel
}

1 Je présente ici mon projet d'étude concernant l'américanisation de la France pendant les années 1950 et $1960^{1}$. Mes travaux s'inscrivent dans le cadre d'une recherche plus large sur le problème de «l'américanisation globale». Un groupe de chercheurs américains, européens et japonais a commencé à analyser ce phénomène, dans sa dimension internationale. Dans cette optique, l'américanisation constitue un changement économique, social et culturel à l'échelle du monde, qui est devenu l'une des caractéristiques qui définissent le $\mathrm{xx}^{\mathrm{e}}$ siècle. Nous considérons que l'américanisation est moins une question d'impérialisme culturel - en partie parce que le phénomène a pris une autonomie croissante à l'égard de l'Amérique - qu'une sorte d'impératif global qui est né en Amérique avant de s'étendre à l'Europe puis au monde entier. Il s'agit donc de définir le phénomène, d'en explorer les variantes, d'expliquer son dynamisme et de déterminer sa signification.

2 Je me suis proposé d'étudier non pas tant l'américanisation que la réaction française - qui va de l'émulation à la résistance - à ce phénomène ou à ce qu'on appelé «le défi américain ». Mon hypothèse est qu'elle a constitué un dilemme pour la France, dans la période qui a suivi la guerre.

3 Comment définir ce terme d'américanisation? Il faisait référence, avant tout, à des niveaux de richesse et de bien-être existant aux USA. Cette notion impliquait non seulement la production et la consommation de produits standardisés, souvent d'origine ou de modèle américain, mais aussi un style de vie qui comportait de nouveaux modèles de dépense, des niveaux de salaire plus élevés, une plus grande mobilité sociale, une vie centrée sur la consommation et une philosophie matérialiste. Elle impliquait également des formes différentes d'organisation et de pratique économiques, par exemple une attention portée aux techniques de la vente. Elle apportait avec elle les produits de la nouvelle culture de masse, tels que les films d'Hollywood, la Sélection du Reader's Digest, et le fast food. 
4 Pour les Français de l'après-guerre, l'Amérique était généralement considérée comme le prototype de la société de consommation à venir. Elle était à la fois un modèle socioéconomique et culturel et une menace. Ma thèse principale est que l'américanisation a constitué un dilemme pour les Français parce qu'elle posait des questions de fond concernant l'indépendance et l'identité de la France. Elle posait la question suivante: Comment les Français peuvent-ils atteindre les niveaux de prospérité que connaissaient les États-Unis, tout en gardant leur identité? En particulier, comment peuvent-ils accepter l'aide économique et les conseils des Américains; emprunter des pratiques économiques américaines; consommer des produits américains; imiter la politique sociale américaine ; s'habiller, lire, parler et, peut-être pire encore, manger comme les Américains, sans perdre leur originalité française ? Pour les Français de l'après-guerre, se lancer dans le consumérisme américain, c'était, semble-t-il, mettre en question l'idée que la nation avait d'elle-même ou son identité, autant que son indépendance vis à vis de l'hégémonie américaine. Pour reprendre les termes d'un journaliste : « Un bistro qui sert du coca au lieu de Beaujolais est-il encore français? »

5 D'un point de vue légèrement différent, l'Amérique de l'après-guerre apparaissait comme un défi, à la fois comme un modèle et une menace, parce que, dans leur effort pour acquérir la prospérité et le pouvoir économique des Américains, les Français voulaient éviter de payer les coûts sociaux et culturels qui y semblaient liés. Le défi était d'acquérir une "modernité » économique tout en évitant les erreurs américaines telles que la conformité sociale, l'agressivité économique, l'uniformité et la stérilité culturelles.

6 Je n'ai pas l'intention cependant, d'analyser toutes les manifestations de l'américanisation. C'est un sujet trop vaste et trop difficile à cerner. Ainsi, je n'étudie pas la façon dont l'Amérique a pu exercer une influence dans des domaines comme l'éducation, la politique, les sciences sociales ou la musique. Je n'essaie pas non plus d'expliquer, en termes strictement économiques, le processus lui-même en l'opposant aux manifestations et aux réactions qu'il a induites. Plutôt que de procéder à un survol général de la question, j'ai choisi de privilégier plusieurs points de rencontre entre la France et l'Amérique, qui s'insèrent au mieux dans mon projet. Chacun d'eux met l'accent sur un point de divergence entre la modernité américaine et la mentalité française. Chacun d'eux est centré sur des groupes sociaux spécifiques, que ce soit le monde des affaires, le monde du travail ou les intellectuels. Considérés dans leur ensemble, ces points de rencontre devraient permettre une compréhension et une explication globales de la réaction française à l'américanisation. Les points de rencontre sont les suivants :

7 - le chapitre 1 analyse ce que l'on appelle « l'incitation à la productivité » lancée sous les auspices du Plan Marshall, au début des années 50. Ce dernier avait pour objectif d'enseigner aux Européens la façon américaine de s'enrichir. Plusieurs milliers d'hommes d'affaires français et de dirigeants syndicaux ont parcouru les États-Unis pour trouver les secrets du niveau de vie élevé du Nouveau Monde.

8 - le chapitre 2 évoque la controverse concernant l'introduction du coca-cola en 1949-1950, qui aboutit aux mesures d'interdiction touchant la plus américaine des boissons, considérée comme un danger pour la santé. Cette controverse donne des indications sur la nature de la résistance des Français à certains produits de consommation américains. Les protagonistes en la circonstance étaient le parti communiste et certains groupes d'intérêts économiques. 
9 - le chapitre 3 analyse la vague d'anti-américanisme qui a sévi au début de la Guerre Froide. Elle a été à l'origine d'une vague de chauvinisme culturel, de manifestations de rues contre la nomination du général Ridgway à l'OTAN et d'attaques contre la politique étrangère américaine. A cette époque, la résistance à l'Amérique débordait largement les rangs des communistes; elle touchait l'ensemble de la classe politique de la $\mathrm{IV}^{\mathrm{e}}$ République, aussi bien que l'opinion publique.

10 - le chapitre 4 présente les débats sur la société de consommation à venir qui agitèrent les intellectuels français pendant les années 50. Les intellectuels de Saint-Germain-desPrés mettaient en garde contre la menace que représentait l'américanisation, insistant sur les dangers de la société de consommation, de la conformité sociale et de la culture de masse.

11 - le chapitre 5 étudie la seconde vague d'anti-américanisme dans les années 60 ; j'ai centré cette étude sur la personnalité du Président de Gaulle, qui constitue une sorte de métaphore pour cette décade. J'y analyserai aussi le débat sur les vertus du mamagement américain, qui a animé le monde des affaires.

12 - le chapitre 6 présente la controverse sur l'afflux des investissements américains dans les années 60. La France fut le seul des pays de l'Europe de l'Ouest à tenter de limiter «l'invasion» des capitaux américains. Dans ce chapitre, les protagonistes sont des hommes politiques gaullistes, des économistes et des hommes d'affaires. A mon sens, pendant cette période, le gaullisme, de façon paradoxale, a exorcisé le démon américain et a conduit à la vague pro-américaine des années 70 et 80 . Je termine cette analyse par un bref épilogue dans lequel j'évoque le glissement intervenu depuis 1970 vers un enthousiasme des Français pour tout ce qui est américain. En fin de compte, la France at'elle ou n'a t'elle pas été américanisée ? C'est à cette question que je m'efforce d'apporter quelques éléments de réflexion dans ma conclusion.

13 J'utilise des sources extrêmement variées. Chaque chapitre exige le recours à différents types de témoignage. Ainsi, le chapitre sur les missions concernant la productivité repose sur les archives des gouvernements français et américains, c'est à dire, les rapports de mission et les archives de l'Agence de la productivité conservés aux Archives Nationales, ainsi que les archives de l'Administration de la Coopération économique (Plan Marshall) à Washington D.C.

14 Le chapitre sur les intellectuels et la société de consommation utilise la littérature populaire concernant l'Amérique ainsi que le revue Esprit et le journal Le Monde. J'ai également dû faire quelques entretiens pour compléter les documents publiés. Bien que ce projet soit loin d'être achevé, il m'est possible, au point où j'en suis, de présenter quelques observations sur le phénomène de l'américanisation de la France de l'aprèsguerre.

15 Tout d'abord, la société de consommation américaine véhiculait un message idéologique, particulièrement pendant les années 50 . L'arrivée de l'Amérique était un phénomène qui comportait de multiples facettes, dont la culture de consommation n'était qu'un aspect. L'Amérique est arrivée en France après la guerre en tant que super-puissance hégémonique, dotée d'un poids militaire, politique et idéologique autant qu'économique. Il est possible que les produits de consommation américains et les produits culturels ne soient pas vraiment devenus disponibles en plus grand nombre pendant les années 50, mais pour les Français, leur présence revêtait une sorte d'aura parce qu'ils semblaient être liés à la nouvelle présence américaine. Ils finirent par être associés à une Amérique 
hégémonique. Ceci se produisit surtout parce qu'on faisait une publicité considérable à la société de consommation américaine. Au temps du Plan Marshall, on vantait «la voie américaine " comme le moyen de venir à bout d'un nombre important de problèmes économiques français et de lutter contre le communisme. L'une des conséquences de cette conjoncture fut que la société de consommation - au moins pour la Gauche - fut étroitement identifiée au capitalisme américain et à l'idéologie anti-idéologique de l'Amérique. Au début des années 50, la Gauche considérait que le coca-cola et les films d'Hollywood participaient d'une « invasion » capitaliste qui véhiculait un message anticommuniste élaboré à Washington.

Deuxièmement, dans les années 50 et 60 , pour certains, et malgré ses succès et l'attraction qu'elle exerçait, l'Amérique constituait un point de référence négatif. La France se distinguait par l'étendue et l'intensité de sa résistance à la politique étrangère des ÉtatsUnis et à l'américanisation. La France se faisait remarquer - au moins parmi les nations de l'Europe de l'Ouest - pour la vigueur de son anti-américanisme. Si, pendant les années 50, les Français ne participèrent qu'à certains aspects des manifestations les plus agressives de cet anti-américanisme, une part importante de l'élite intellectuelle et politique et au moins un groupe socio-politique, en l'occurrence ceux qui soutenaient le Parti communiste, invitait à résister à tout ce que représentait l'Amérique, y compris la société de consommation. Au cours des années 60, le Président De Gaulle mena une offensive diplomatique qui mérite le nom d'«anti-américaine» et encouragea une seconde vague d'hostilité qui comportait des manifestations de chauvinisme culturel. Mais l'attaque gaulliste était en grande partie une affaire de politique internationale, et contribua, par une sorte de paradoxe, à affaiblir l'anti-américanisme.

D'une manière générale, l'anti-américanisme avait pour origine des problèmes de politique internationale. Au cours des années 50, il procédait de l'opposition de la France au zèle que mettait l'Amérique à combattre le communisme; à la présence de bases militaires américaines; à la politique étrangère de Washington, par exemple en ce qui concernait l'Allemagne et l'Empire français, et à l'ingérence de Washington dans les affaires de la France. La tension culmina en 1952-53, au moment de la défaite de la Communauté Européenne de Défense, et en 1956, lors de l'affaire de Suez. L'antiaméricanisme procédait aussi d'une perte de prestige de la France sur la scène internationale et de l'état de dépendance, qu'elle acceptait mal, dans lequel elle se trouvait. L'ascension de l'Amérique au rang de puissance mondiale et le recul de la France au rang de puissance secondaire semblaient aller de pair-comme si, en quelque sorte, l'une découlait de l'autre. Un tel changement de statut n'était pas facile à accepter pour une nation qui pendant longtemps avait joué le rôle d'une grande puissance et se considérait en outre comme supérieure, sur le plan de la culture, à son nouveau protecteur et mentor d'outre-Atlantique, qui ne s'autorisait que de lui-même. Pour la France, ce nouvel ascendant qu'exerçait l'Amérique d'après-guerre avait vaguement un air $\mathrm{d}^{\prime}$ ' impérialisme »; ce dernier se manifesta très clairement dans les années 60 , avec la Guerre du Viet-Nam et l'afflux des dollars vers l'Europe.

Troisièmement - et c'est là une question plus pointue - peut-on rendre compte de la résistance de la France à la culture de consommation en termes d'anti-américanisme? Cette résistance - conduite par les intellectuels mais qui ne se limitait pas à ces derniers peut être attribuée essentiellement à la crainte que la culture de masse et le consumérisme américains fassent courir un risque à l'identité nationale, autant qu'à la phobie de voir le consumérisme détruire le consensus social. Ce qui suscitait cette 
opposition, c'était la crainte que l'américanisation signifie conformité sociale, uniformisation culturelle, et suppression de la supériorité française et de son caractère unique. Cette fierté de la spécificité française a été renforcée du fait de la période douloureuse de 1940-1945, qui s'acheva avec la libération de la France par les forces anglo-américaines. Pendant les années 50, la dépendance vis à vis des États-Unis en ce qui concerne l'assistance économique et la protection militaire, et la popularité des produits culturels tels que la Sélection du Reader's Digest, ont aiguisé cette détermination à maintenir un sentiment de fierté nationale. Ce qui était en danger dans le processus d'américanisation, c'était l'identité française que les intellectuels plaçaient très haut et identifiaient à la civilisation. Les Français, ceux qui n'appartenaient pas à l'élite, avaient des sentiments ambivalents vis à vis de la politique étrangère américaine des années 50 et ne se préoccupaient guère, ni de la culture de masse américaine, ni de ses produits de consommation. Grâce à des sondages d'opinion effectués au début des années 50, nous disposons de données concernant les sentiments profonds vis à vis de l'Amérique et de l'américanisation. Ces enquêtes montrent que, dans l'ensemble, les Français considéraient l'Amérique comme une force constructive en Europe. Les États-Unis œuvraient à la paix, à la liberté et au progrès socio-économique. Cependant la politique étrangère américaine était aussi fortement considérée comme une menace pour l'indépendance de la France. La présence américaine, par exemple les bases américaines et les films, était tolérée plutôt que désirée - deux personnes sur trois souhaitaient que l'influence de l'Amérique diminue. Et les Américains étaient généralement considérés comme de grands enfants - stéréotype qui avait des connotations aussi bien positives que négatives. Toutefois, peu de ces personnes considéraient l'américanisation comme une menace culturelle. Il est clair que le danger culturel était plus une perception de l'élite que du peuple. En fin de compte, le Français moyen accueillait favorablement la plupart des produits de consommation américains, tels que les appareils ménagers, la Sélection du Reader's Digest, les cigarettes, et même les conserves. Toutefois, le chewing-gum et le cocacola n'attiraient qu'une infime minorité. Les opinions concernant les films américains et le jazz étaient extrêmement partagées. Les produits de consommation et la culture de masse suscitaient des réactions divergentes auprès de l'opinion publique et de l'élite intellectuelle.

19 Quatrièmement, en termes strictement économiques, l'américanisation a correspondu à une étape de la modernisation économique de la France. Elle a fait partie de la révolution qui a affecté les habitudes de consommation. Vers le milieu des années 50, la France s'était engagée dans la période de croissance économique la plus soutenue de l'époque moderne - croissance due en grande partie à l'augmentation de la demande de consommation. Les niveaux de revenus et les modèles de consommation connurent un grand bouleversement. La priorité des dépenses dans le budget des ménages allait à l'équipement de la maison; venaient ensuite l'acquisition d'une automobile et enfin les dépenses de loisir et les dépenses culturelles. Les dépenses concernant l'équipement de la maison augmentèrent plus rapidement en France que dans les autres pays européens - à l'exception de l'Allemagne de l'Ouest. Les dépenses concernant les appareils ménagers augmentèrent de $15 \%$ par an, avec une accélération particulièrement vive après 1954 . Beaucoup de ces produits étaient fabriqués par des sociétés américaines et certains portaient des marques comme Frigidaire et Kodak. L'américanisation, sous sa forme de production et d'achat de produits de consommation et d'élévation du niveau de vie, fut à la fois un facteur important de croissance économique et un indice de cette croissance. 

années 50. Au cours de cette décennie, en gros, les États-Unis ont aidé la IV République à trouver une légitimité, et à tenir à l'écart les communistes et les gaullistes. Les gouvernements français et américain unirent leurs efforts (malgré l'inconsistance de la IV ${ }^{\mathrm{e}}$ République) pour présenter la prospérité de type américain comme un bon moyen de lutter contre le communisme. Au cours de la décennie qui suivit, le Président De Gaulle lutta contre l'hégémonie américaine en Europe de l'Ouest, mais l'américanisation progressa dans le pays, en dépit de quelques controverses mineures concernant des questions comme celle des investissements américains; cette progression fut en partie due à la politique économique gaulliste. Au cours des années 60, malgré De Gaulle, ou peut-être grâce à lui, le problème de l'américanisation ne fut plus qu'une question de politique intérieure. été utilisée dans la lutte contre le communisme sur la scène politique intérieure ; qu'elle a fortement contribué à la modernisation économique; qu'elle a nourri l'affirmation culturelle et animé la controverse sur l'identité nationale. Toutefois, la résistance à l'américanisation et l'anti-américanisme ont diminué en même temps que les groupes d'opposition militante s'affaiblissaient et se transformaient, et que la société de consommation était progressivement adoptée. Au fur et à mesure que les voix politiques les plus fortes (communistes et gaullistes) s'affaiblissaient et que les intellectuels de gauche évoluaient, l'antagonisme s'atténuait. La résistance diminua lorsque la France eut atteint une certaine indépendance et se résigna à n'avoir qu'une place de moyenne importance dans les affaires du monde. Les perceptions s'adoucirent lorsque le consumérisme s'installa sans bousculer les habitudes françaises. Puis, à un certain moment, la société de consommation fut dissociée de l'Amérique. On en vint à reconnaître que l'Amérique ne commettait pas quelque crime culturel, mais qu'elle était la co-victime ou la co-bénéficiaire - selon la manière de voir - d'un impératif économique global.

\section{NOTES}

1. Après cinq années de travail, la rédaction de l'ouvrage est presque achevée. J'ai bénéficié de bourses de la Fondation Marshall aux États-Unis, du Centre international des Boursiers Woodrow Wilson et de la Fondation John Simon Guggenheim. L'EHESS m'a donné la possibilité, au printemps 1989, de compléter mes recherches à Paris à l'occasion d'un mois d'enseignement comme directeur d'études associé. 


\section{AUTEUR}

RICHARD KUISEL

Richard KUISEL est professeur à la State University of New York à Stone Brook. 